\title{
The black holes of topologically massive gravity
}

\author{
Karim Ait Moussa ${ }^{a, b}{ }^{*}$, Gérard Clément ${ }^{a}{ }^{\dagger}$ and Cédric Leygnac ${ }^{a} \ddagger$ \\ ${ }^{a}$ Laboratoire de Physique Théorique LAPTH (CNRS), \\ B.P.110, F-74941 Annecy-le-Vieux cedex, France \\ ${ }^{b}$ Laboratoire de Physique Mathématique et Physique Subatomique, \\ Département de Physique, Faculté des Sciences, Université Mentouri, \\ Constantine 25000, Algeria
}

October 29, 2018

\begin{abstract}
We show that an analytical continuation of the Vuorio solution to three-dimensional topologically massive gravity leads to a two-parameter family of black hole solutions, which are geodesically complete and causally regular within a certain parameter range. No observers can remain static in these spacetimes. We discuss their global structure, and evaluate their mass, angular momentum, and entropy, which satisfy a slightly modified form of the first law of thermodynamics.
\end{abstract}

\footnotetext{
*Email: karim.aitmoussa@wissal.dz

${ }^{\dagger}$ Email: gclement@lapp.in2p3.fr

${ }^{\ddagger}$ Email: leygnac@lapp.in2p3.fr
} 
It is well known that Einstein gravity in $2+1$ dimensions is dynamically trivial, without propagating degrees of freedom. The addition to the Einstein-Hilbert action of a gravitational Chern-Simons term leads to topologically massive gravity [1] (TMG), with massive spin 2 excitations. A long standing question 2] is that of the existence of Schwarzschild- or Kerrlike black hole solutions to TMG. The equations of TMG with a negative cosmological constant are trivially solved [3] by the BTZ black-hole metric 4. Other black hole solutions to cosmological TMG were written down in [5. 6], however it is possible to show that these solutions are not bona fide black holes for the range of parameters considered in [5, 6]. A number of exact solutions to TMG with vanishing cosmological constant are known 17, 8, 9, 10, but these do not include black hole solutions. The purpose of this Letter is to show that a simple extension of the Vuorio solution [7] leads to regular TMG black holes, and to present an introductory exploration of their properties.

The field equations of TMG are

$$
G^{\mu}{ }_{\nu}+\frac{1}{\mu} C_{\nu}^{\mu}=0
$$

where $G^{\mu}{ }_{\nu} \equiv R^{\mu}{ }_{\nu}-\frac{1}{2} R \delta^{\mu}{ }_{\nu}$ is the Einstein tensor,

$$
C_{\nu}^{\mu} \equiv \varepsilon^{\mu \alpha \beta} D_{\alpha}\left(R_{\beta \nu}-\frac{1}{4} g_{\beta \nu} R\right)
$$

is the Cotton tensor (the antisymmetrical tensor is $\varepsilon^{\mu \alpha \beta}=|g|^{-1 / 2} \eta^{\mu \alpha \beta}$, with $\eta^{012}=+1$ ), and $\mu$ is the topological mass constant. Vuorio searched for stationary rotationally symmetric solutions to these equations, and noticed that they could be solved exactly by assuming a constant $g_{t t}$, which he normalized to the Minkowski value ${ }^{1} g_{t t}=-1$. Vuorio's solution is, in units such that $\mu=+3$,

$$
d s^{2}=-[d \tilde{t}-(2 \cosh \sigma+\tilde{\omega}) d \tilde{\varphi}]^{2}+d \sigma^{2}+\sinh ^{2} \sigma d \tilde{\varphi}^{2},
$$

where $\tilde{\varphi}$ is assumed to be periodic with period $2 \pi$, and we have reintroduced an integration constant $\tilde{\omega}$ which Vuorio set to -2 for regularity. This spacetime is homogeneous, with constant curvature scalars, and admits four Killing vectors generating the Lie algebra of $S L(2, R) \times U(1)$ [7. 11. However, it has one undesirable property. From (33) we obtain

\footnotetext{
${ }^{1}$ Contrary to Vuorio, we use the signature $(-++)$ for a Lorentzian metric.
} 
$g_{\tilde{\varphi} \tilde{\varphi}}=-3 \cosh ^{2} \sigma+4 \tilde{\omega} \cosh \sigma-\tilde{\omega}^{2}-1$, which is negative definite if $\tilde{\omega}^{2}<3$, and negative outside a critical radius $\sigma_{c}$ if $\tilde{\omega}^{2}>3$, leading to closed timelike circles $^{2}$ for all $\sigma>\sigma_{c}$.

To overcome this indesirable violation of causality, let us analytically continue the solution (3) by the combined imaginary coordinate transformation (which does not change the overall Lorentzian signature) $\tilde{t}=i \sqrt{3} t$, $\tilde{\varphi}=i\left(\rho_{0} / \sqrt{3}\right) \varphi$, with $\rho_{0}$ an arbitrary positive constant, leading to

$$
d s^{2}=3\left[d t-\left(\frac{2 \rho}{3}+\omega\right) d \varphi\right]^{2}+\frac{d \rho^{2}}{\rho^{2}-\rho_{0}^{2}}-\frac{\rho^{2}-\rho_{0}^{2}}{3} d \varphi^{2},
$$

where $\omega=\rho_{0} \tilde{\omega} / 3$, the new radial coordinate is $\rho=\rho_{0} \cosh \sigma$, and $\varphi$ is again assumed to be an angular variable with period $2 \pi$. This new solution of the field equations can also be derived directly à la Vuorio by making the unconventional ansatz $g_{t t}=+3$ (instead of -1 ). The fact that the Killing vector field $\partial_{t}$ is spacelike, not timelike, means that there can be no static observers in such a geometry. Furthermore it is easily seen that no linear combination of the Killing vectors $\partial_{t}$ and $\partial_{\varphi}$ can remain timelike for $\rho \rightarrow \infty$. This situation is quite similar to that inside the ergosphere of the Kerr metric, except that here the ergosphere extends to spacelike infinity. As in the case of the Kerr ergosphere, the solution is however locally stationary. At a given radius $\rho$, observers moving with uniform angular velocity $\Omega$ remain timelike, $\left(\partial_{t}+\Omega \partial_{\varphi}\right)^{2}<0$, provided

$$
\frac{2 \rho+3 \omega-\sqrt{\rho^{2}-\rho_{0}^{2}}}{r^{2}}<\Omega<\frac{2 \rho+3 \omega+\sqrt{\rho^{2}-\rho_{0}^{2}}}{r^{2}} .
$$

The divergence of the metric component $g_{\rho \rho}$ at $\rho= \pm \rho_{0}$ suggests that (4) is a black hole solution, which is made obvious by rearranging the metric as

$$
d s^{2}=-\frac{\rho^{2}-\rho_{0}^{2}}{r^{2}} d t^{2}+\frac{d \rho^{2}}{\rho^{2}-\rho_{0}^{2}}+r^{2}\left(d \varphi-\frac{2 \rho+3 \omega}{r^{2}} d t\right)^{2}
$$

with

$$
r^{2}=\rho^{2}+4 \omega \rho+3 \omega^{2}+\rho_{0}^{2} / 3 .
$$

While this metric is not asymptotically Minkowskian, the squared lapse $N^{2}=\left(\rho^{2}-\rho_{0}^{2}\right) / r^{2}$ and the shift $N^{\varphi}=-(2 \rho+3 \omega) / r^{2}$ respectively go to 1

\footnotetext{
${ }^{2}$ The same is true for the purported black-hole solutions of [5] with $\Lambda=0,2 J>M$, or of [6] with $\lambda=0, a_{0}>0$.
} 
and 0 at spacelike infinity $\rho \rightarrow \pm \infty$. For $\rho_{0}^{2}>0$, there are two horizons located at $\rho_{h \pm}= \pm \rho_{0}$, of perimeter and angular velocity

$$
A_{h \pm} \equiv 2 \pi r_{h \pm}=2 \pi\left|2 \rho_{0} \pm 3 \omega\right| / \sqrt{3}, \quad \Omega_{h \pm}=3 /\left( \pm 2 \rho_{0}+3 \omega\right) .
$$

If $\omega \neq \mp 2 \rho_{0} / 3$, the metric may be extended through these horizons by the usual Kruskal method. Just as in the case of the BTZ black holes, the metric (4) is regular for all $\rho \neq \pm \rho_{0}$, so that the maximally extended spacetime is geodesically complete, with a Penrose diagram similar to that of the Kerr black hole (except of course for the ring singularity). However, singularities in the causal structure do occur for a range of values of $\omega$. If $\omega^{2}<\rho_{0}^{2} / 3$, the Killing vector $\partial_{\varphi}$ is everywhere spacelike. On the contrary, if $\omega^{2}>\rho_{0}^{2} / 3, \partial_{\varphi}$ becomes timelike in the range $\rho_{c-}<\rho<\rho_{c+}$, with

$$
\rho_{c \pm}=-2 \omega \pm \sqrt{\omega^{2}-\rho_{0}^{2} / 3}
$$

It is easily seen that these two zeros of $\left(\partial_{\varphi}\right)^{2}$ are timelike lines belonging to the same stationary Kruskal patch, so that the acausal regions $r^{2}<0$ are safely hidden behind the horizon for an observer at $\rho=+\infty$ if $\omega>0$, which we shall assume henceforth. The Penrose diagram for the maximally extended spacetime with the acausal regions cut out is the same as for Reissner-Nordström black holes. The limiting case $\rho_{0}=0$ leads to extreme black holes, with a double horizon at $\rho=0$. In this case, there is an acausal region $\left(\partial_{\varphi}\right)^{2}<0$ behind the horizon for all positive values of $\omega$. The resulting Penrose diagram (again with the acausal regions cut out) is identical to that of extreme Reissner-Nordström black holes.

The case $\omega=2 \rho_{0} / 3$ deserves special consideration. In this case the metric ([6) reduces to

$$
d s^{2}=-\frac{\rho-\rho_{0}}{\rho+5 \rho_{0} / 3} d t^{2}+\frac{d \rho^{2}}{\rho^{2}-\rho_{0}^{2}}+\left(\rho+5 \rho_{0} / 3\right)\left(\rho+\rho_{0}\right)\left(d \varphi-\frac{2 d t}{\rho+5 \rho_{0} / 3}\right)^{2} .
$$

This has only one horizon at $\rho=+\rho_{0}$, where Kruskal extension can be carried out as usual. Near the causal singularity $\rho=-\rho_{0}$, the metric (10) can be approximated by

$$
d s^{2} \simeq 3 d t^{2}+d \sigma^{2}, \quad d \sigma^{2}=-\frac{d \rho^{2}}{2 \rho_{0}\left(\rho+\rho_{0}\right)}+\left(2 \rho_{0} / 3\right)\left(\rho+\rho_{0}\right) d \hat{\varphi}^{2}
$$

with $d \hat{\varphi}=d \varphi-3 d t$. Clearly the two-dimensional metric $d \sigma^{2}$ becomes null for $\rho \rightarrow-\rho_{0}$, and is non-extendible because of the periodicity condition on $\varphi$, so 
that $\rho=-\rho_{0}$ is a spacelike singularity of the metric (10). The corresponding Penrose diagram is thus identical to that of the Schwarzschild black hole.

The even more special case $\omega=\rho_{0}=0$ lies at the intersection of the preceding case and of the extreme black hole case $\rho_{0}=0$. In this case the metric (6) reduces to

$$
d s^{2}=-d t^{2}+\frac{d \rho^{2}}{\rho^{2}}+\rho^{2}\left(d \varphi-\frac{2}{\rho} d t\right)^{2},
$$

which is devoid of horizons, and thus qualifies as the ground state or "vacuum" of our two-parameter family of black-hole solutions. This metric does not appear to be extendible beyond the manifest singularity $\rho=0$, which can be shown to be at finite affine distance. Moreover, the angular velocity of stationary observers approaching this singularity increases without bound, so that the very concept of a Penrose diagram breaks down.

The black hole metric (6) depends on two parameters $\rho_{0}$ and $\omega$, which should somehow be related to the physical parameters, mass and angular momentum. The standard approach for computing these quantities in the case of non-asymptotically flat spacetimes ${ }^{3}$ uses the idea of quasilocal energy 13. From the action functional for a self-gravitating system with boundary conditions on a given hypersurface, one derives canonically a Hamiltonian, given by the sum of a bulk integral, which vanishes on shell, and of a surface term. The quasilocal energy is the (substracted) on-shell value of the Hamiltonian in the limit where the spatial boundary is taken to infinity. A canonical formulation of topologically massive gravity was given in [14]. However integrations by part were freely performed in [14, so that at present we do not know what is the correct surface term. Instead we shall bypass the standard quasilocal approach by suitably extending the recently proposed super angular momentum approach to the computation of conserved quantities in $2+1$ gravity [15. This approach relies on the observation that the dimensional reduction of a self-gravitating system with two Killing vectors $\partial_{t}$ and $\partial_{\varphi}$ leads to a mechanical system with the $S L(2, R) \sim S O(2,1)$ invariance. This mechanical system has a conserved super angular momentum, two components of which may be identified as the mass and angular momentum of the $(2+1)$-dimensional gravitating configuration. These identifications have been shown in [15] to correspond to a well-defined "finite part" prescription for computing the (unsubstracted) quasilocal conserved

\footnotetext{
${ }^{3}$ The computation of energy and angular momentum for asymptotically Minkowkian or asymptotically (A)dS solutions to TMG recently carried out in 12 cannot be used here.
} 
quantities, and to lead to consistent results for Einstein-scalar and (up to some gauge ambiguity) for Einstein-Maxwell black holes.

The conserved super angular momentum for TMG has been given in [10]. The general stationary rotationally symmetric metric may be written in the $2+1$ form

$$
d s^{2}=\lambda_{a b}(\rho) d x^{a} d x^{b}+\zeta^{-2}(\rho) R^{-2}(\rho) d \rho^{2},
$$

where $\lambda$ is the $2 \times 2$ matrix

$$
\lambda=\left(\begin{array}{cc}
T+X & Y \\
Y & T-X
\end{array}\right)
$$

$R^{2}=\mathbf{X}^{2}=-T^{2}+X^{2}+Y^{2}$ is the Minkowski pseudo-norm of the vector $\mathbf{X}(\rho)=(T, X, Y)$, and the function $\zeta(\rho)$ allows for arbitrary reparametrizations of the radial coordinate $\rho$. In the gauge $\zeta=1$, the conserved generalized angular momentum for TMG is

$$
\mathbf{J}=\frac{1}{2 \kappa}\left(\mathbf{X} \wedge \mathbf{X}^{\prime}+\frac{1}{2 \mu}\left[\mathbf{X}^{\prime} \wedge\left(\mathbf{X} \wedge \mathbf{X}^{\prime}\right)-2 \mathbf{X} \wedge\left(\mathbf{X} \wedge \mathbf{X}^{\prime \prime}\right)\right]\right)
$$

where $\kappa=8 \pi G$ is the Einstein gravitational constant, the prime is the derivative $d / d \rho$, and the wedge product is defined by $(\mathbf{X} \wedge \mathbf{Y})^{A}=$ $\eta^{A B} \epsilon_{B C D} X^{C} Y^{D}$ (with $\eta^{A B}$ the inverse Minkowski metric, and $\epsilon_{012}=+1$ ). Assuming that the identifications of Einstein-scalar or Einstein-Maxwell black hole conserved quantities proposed in [15] can be extended to TMG, the $(2+1)$-dimensional mass and angular momentum are given by

$$
\begin{aligned}
M & =-2 \pi J^{Y}, \\
J & =2 \pi\left(J^{T}-J^{X}\right) .
\end{aligned}
$$

We first test these formulas on the example of the BTZ solution, for which the quasilocal mass and angular momentum have recently been computed in the wider framework of a Poincaré gauge theory [16] (the action for this theory reduces to that of TMG when a constraint for vanishing torsion is added). The BTZ metric is given by

$$
\begin{aligned}
d s^{2}= & \left(-2 l^{-2} \rho+\mathrm{M} / 2\right) d t^{2}-\mathrm{J} d t d \varphi+\left(2 \rho+\mathrm{M} l^{2} / 2\right) d \varphi^{2} \\
& +\left[4 l^{-2} \rho^{2}-\left(\mathrm{M}^{2} l^{2}-\mathrm{J}^{2}\right) / 4\right]^{-1} d \rho^{2},
\end{aligned}
$$

with $\Lambda=-l^{-2}$ the cosmological constant. The parametrization (14) of this metric corresponds to 15

$$
\mathbf{X}=\mid \begin{aligned}
& \left(1-l^{-2}\right) \rho+\left(1+l^{-2}\right) \mathrm{M} l^{2} / 4 \\
& -\left(1+l^{-2}\right) \rho-\left(1-l^{-2}\right) \mathrm{M} l^{2} / 4 \\
& -\mathrm{J} / 2
\end{aligned},
$$


with $\zeta=1$. The computation of the superangular momentum (15) is straightforward and gives the TMG mass and angular momentum of the BTZ black holes in terms of the Einstein conserved quantities $\mathrm{M}$ and J,

$$
M=\frac{\pi}{\kappa}\left(\mathrm{M}-\frac{\mathrm{J}}{\mu l^{2}}\right), \quad J=\frac{\pi}{\kappa}\left(\mathrm{J}-\frac{\mathrm{M}}{\mu}\right) .
$$

These values (which reduce to the Einstein values in the limit $\mu \rightarrow \infty$ ) coincide with the values (22) and (23) obtained in [16] in the special case of a vanishing torsion $\mathcal{T}=0$ (the identification is $2 \ell \theta_{L}=-1 / \mu, \Lambda_{\text {eff }}=-\Lambda=$ $\left.l^{-2}, \ell=\kappa=\pi, \chi=1\right)$.

Strengthened by this agreement, we proceed with the computation of the conserved quantities $M$ and $J$ for the $\Lambda=0$ TMG black holes. The parametrization (14) of the metric (6) corresponds to

$$
\mathbf{X}=\mid \begin{aligned}
& \rho^{2} / 2+2 \omega \rho+3\left(\omega^{2}+1\right) / 2+\rho_{0}^{2} / 6 \\
& -\rho^{2} / 2-2 \omega \rho-3\left(\omega^{2}-1\right) / 2-\rho_{0}^{2} / 6 \\
& -2 \rho-3 \omega
\end{aligned},
$$

with $\zeta=1$. The computation of the super angular momentum (15) leads to

$$
M=\frac{\pi}{\kappa} \omega, \quad J=\frac{\pi}{\kappa}\left(\omega^{2}-5 \rho_{0}^{2} / 9\right) .
$$

Surprisingly, the mass depends only on the parameter $\omega$, and not on the ostensible horizon radius $\rho_{0}$. These relations can be inverted to yield

$$
\omega=\frac{\kappa}{\pi} M, \quad \rho_{0}^{2}=\frac{9 \kappa^{2}}{5 \pi^{2}}\left(M^{2}-\pi J / \kappa\right) .
$$

Extreme black holes thus have $J=\kappa M^{2} / \pi$, Schwarzschild-like black holes have $J=-\kappa M^{2} / 4 \pi$, while the mass and angular momentum vanish for the vacuum solution (12), as expected.

What are the thermodynamical properties of these black holes? The Hawking temperature depends only on the metric, not on the particular theory of gravity considered, and is given by the inverse of the period in imaginary time,

$$
\left.T_{H} \equiv \frac{1}{2 \pi} n^{\rho} \partial_{\rho} N\right|_{\left(\rho=\rho_{h}\right)}=\left.\frac{\zeta R R^{\prime}}{2 \pi \sqrt{V}}\right|_{\left(\rho=\rho_{h}\right)} .
$$

We obtain here the temperature

$$
T_{H}=\frac{\sqrt{3}}{2 \pi} \frac{\rho_{0}}{2 \rho_{0}+3 \omega}
$$


which as usual vanishes for extreme black holes $\left(\rho_{0}=0\right)$. On the other hand, the black hole entropy should depend on the specific theory under consideration, and we have no reason to expect that it is given by the Einstein value $S_{E}=(2 \pi / \kappa) A_{h}$. To determine the black hole entropy, we use the first law of thermodynamics, according to which the entropy variation is given by

$$
\left.\frac{\partial S}{\partial M}\right|_{J}=T_{H}^{-1}
$$

This can be integrated to yield

$$
S=\frac{2 \pi^{2}}{3 \sqrt{3} \kappa}\left(5 \rho_{0}+6 \omega\right),
$$

up to an arbitrary additive function of $J$. Assuming that this function vanishes, we obtain from (27), (25), (22) and (8) a modified form of the first law of black hole thermodynamics:

$$
d M=T_{H} d S+\frac{1}{2} \Omega_{h} d J .
$$

The anomalous factor $1 / 2$ in front of the angular velocity is another surprising effect of TMG. Finally, a simple quadratic combination of the same (undifferentiated) quantities yields the Smarr-like formula

$$
M=T_{H} S+\Omega_{h} J
$$

to be compared with the Smarr-like formula for $2+1$ Einstein-scalar black holes [15] $M=T_{H} S / 2+\Omega_{h} J$.

A problem with the value (22) for the black hole mass is that, contrary to the case of four-dimensional Einstein gravity, the gravitational constant $\kappa$ must be negative in TMG to avoid the occurrence of ghosts [1]. This means that, for $\omega^{2}>\rho_{0}^{2} / 3$, causally regular black holes, with $\omega>0$, have a negative mass (as well as a negative entropy). For $\omega^{2}<\rho_{0}^{2} / 3$, all black holes are regular, but only those with $\omega<0$ have a positive mass (but a negative entropy). We do not know how to solve this problem, but point out that a similar problem arises with the BTZ black holes viewed as solutions of TMG with negative gravitational constant. It follows from (20) that, for $\kappa<0$, regular BTZ black holes, with $\mathrm{M}>0$ and $\mathrm{J}^{2} \leq \mathrm{M}^{2} l^{2}$, necessarily have a negative TMG mass $M$ if $\mu^{2} l^{2}>1$, and do not necessarily have a positive TMG mass if $\mu^{2} l^{2} \leq 1$.

We have shown that an analytical extension of the Vuorio solution to TMG leads to a two-parameter family of black hole solutions, which are 
causally regular within a certain parameter range. While their metric is not asymptotically Minkowskian, the AdM lapse and shift functions go to the Minkowski values 1 and 0 at spacelike infinity, so that their Penrose diagrams are similar to those of Kerr or Reissner-Nordström black holes. In this respect, these black hole spacetimes are closer to four-dimensional black holes than the asymptotically AdS BTZ black holes of 4. No observers can remain static in these spacetimes, however stationary observers are allowed, which is all that is needed to discusss physical experiments such as wave scattering. We have evaluated the mass, angular momentum, and entropy of these black holes, which satisfy a slightly modified form of the first law of thermodynamics. At present these evaluations remain tentative. Our formulas (16) and (17) for computing the black hole mass and angular momentum generalize formulas previously derived and tested in the case of Einstein-scalar or Einstein-Maxwell black holes, and have been tested here in the specific case of the BTZ solution to cosmological TMG. However a full computation of the quasilocal energy and angular momentum in TMG should be carried out in order to ascertain the validity of our evaluations (the computations of [16 cannot be adapted for this purpose because the field equations of the Poincaré gauge theory considered there are stronger than those of TMG, and do not admit our black holes as solutions). A direct computation of the black hole entropy is also desirable. We intend to address these questions, and to further elucidate the properties of our black holes, in a future publication.

\section{References}

[1] S. Deser, R. Jackiw and S. Templeton, Phys. Rev. Lett. 48 (1982) 975; Ann. Phys., NY 140 (1982) 372.

[2] S. Deser, private communication.

[3] N. Kaloper, Phys. Rev. D 48 (1993) 2598.

[4] M. Bañados, C. Teitelboim and J. Zanelli, Phys. Rev. Lett. 69 (1992) 1849; M. Bañados, M. Henneaux, C. Teitelboim and J. Zanelli, Phys. Rev. D 48 (1993) 1506.

[5] Y. Nutku, Class. Quant. Grav. 10 (1993) 2657.

[6] M. Gürses, Class. Quant. Grav. 11 (1994) 2585. 
[7] I. Vuorio, Phys. Lett. 163B (1985) 91; R. Percacci, P. Sodano and I. Vuorio, Ann. Phys., NY 176 (1987) 344.

[8] G.S. Hall, T. Morgan and Z. Perjès, Gen. Rel. Grav. 19 (1987) 1137.

[9] Y. Nutku and P. Baekler, Ann. Phys., NY 195 (1989) 16.

[10] G. Clément, Class. Quantum Grav. 11 (1994) L115.

[11] M.E. Ortiz, Ann. Phys, NY 200 (1990) 345.

[12] S. Deser and B. Tekin, Class. Quantum Grav. 20 (2003) L259.

[13] J.D. Brown and J.W. York, Phys. Rev. D 47 (1993) 1407.

[14] S. Deser and X. Xiang, Phys. Lett. B 263 (1991) 39.

[15] G. Clément, Phys. Rev. D 68 (2003) 024032.

[16] A.A. García, F.W. Hehl, C. Heinicke and A. Macías, Phys. Rev. D 67 (2003) 124016. 